The Journal of Laryngology \& Otology

http://journals.cambridge.org/JLO

Additional services for The Journal of Laryngology \& Otology:

Email alerts: $\underline{\text { Click here }}$

Subscriptions: $\underline{\text { Click here }}$

Commercial reprints: Click here

Terms of use : $\underline{\text { Click here }}$

\title{
Effect of cochlear implant electrode insertion on middle-ear function as measured by intra-operative laser Doppler vibrometry
}

N Donnelly, A Bibas, D Jiang, D-E Bamiou, C Santulli, G Jeronimidis and A Fitzgerald O'Connor

The Journal of Laryngology \& Otology / Volume 123 / Issue 07 / July 2009, pp 723 - 729

DOI: 10.1017/S0022215109004290, Published online: 13 January 2009

Link to this article: http://journals.cambridge.org/abstract_S0022215109004290

How to cite this article:

N Donnelly, A Bibas, D Jiang, D-E Bamiou, C Santulli, G Jeronimidis and A Fitzgerald O'Connor (2009). Effect of cochlear implant electrode insertion on middle-ear function as measured by intra-operative laser Doppler vibrometry. The Journal of Laryngology \& Otology, 123, pp 723-729 doi:10.1017/S0022215109004290

Request Permissions : $\underline{\text { Click here }}$ 


\title{
Effect of cochlear implant electrode insertion on middle-ear function as measured by intra-operative laser Doppler vibrometry
}

\author{
N Donnelly*, A Bibas*†, D Jiang*, D-E Bamiou $\neq$, C Santulli**, G Jeronimidis**, \\ A FitzGerald O'CONNOR*
}

\begin{abstract}
Hypothesis: The aim of this study was to investigate the impact of cochlear implant electrode insertion on middle-ear low frequency function in humans.

Background: Preservation of residual low frequency hearing with addition of electrical speech processing can improve the speech perception abilities and hearing in noise of cochlear implant users. Preservation of low frequency hearing requires an intact middle-ear conductive mechanism in addition to intact inner-ear mechanisms. Little is known about the effect of a cochlear implant electrode on middle-ear function.

Methods: Stapes displacement was measured in seven patients undergoing cochlear implantation. Measurements were carried out intra-operatively before and after electrode insertion. Each patient acted as his or her own control. Sound was delivered into the external auditory canal via a speaker and calibrated via a probe microphone. The speaker and probe microphone were integrated into an individually custom-made ear mould. Ossicular displacement in response to a multisine stimulus at $80 \mathrm{~dB}$ SPL was measured at the incudostapedial joint via the posterior tympanotomy, using an operating microscope mounted laser Doppler vibrometry system.

Results: Insertion of a cochlear implant electrode into the scala tympani had a variable effect on stapes displacement. In three patients, there was little change in stapes displacement following electrode insertion. In two patients, there was a significant increase, while in a further two there was a significant reduction in stapes displacement. This variability may reflect alteration of cochlear impedance, possibly due to differing loss of perilymph associated with the electrode insertion.

Conclusion: Insertion of a cochlear implant electrode produces a change in stapes displacement at low frequencies, which may have an effect on residual low frequency hearing thresholds.
\end{abstract}

Key words: Cochlear Implant; Middle Ear; Physiology; Laser Doppler Vibrometry

\section{Introduction}

In the newest application of neural prostheses for deafness, electrical stimulation from a cochlear implant is used to complement residual, low frequency hearing. Using the technique of 'soft surgery', electrodes are inserted into the scala tympani with the hope of preserving neural elements in the apical region of the cochlea. When hearing is preserved, the combination of acoustic hearing in low frequencies $(125-750 \mathrm{~Hz})$ and electrical stimulation of high frequencies (termed electric-acoustic stimulation) can lead to very high levels of speech understanding, especially in noise. ${ }^{1-5}$ Additionally, an appreciation of music and of the quality of sound is maintained, due to residual hearing providing finer spectral resolution than is possible with current cochlear implant speech-processing algorithms. ${ }^{6}$

One of the central issues in electric-acoustic stimulation is the depth of the electrode insertion and its relationship with speech understanding. Improvement of speech discrimination scores has been reported for both short distance $(10 \mathrm{~mm})$ and longer distance $(20 \mathrm{~mm})$ insertion depths. ${ }^{1,7}$ Two obvious concerns exist for longer distance insertion. One is the realistic risk of injury to remaining hair cells; the other is the potential modification of cochlear impedance, which may lead to altered

From the *Auditory Implantation Unit, Department of Otolaryngology, Head and Neck Surgery, Guy's and St Thomas' Hospitals, London, UK, the $†$ Department of Otolaryngology - Head and Neck Surgery, Hippokrateion Hospital, University of Athens Medical School, Greece, the $\ddagger$ Department of Neurotology, Institute of Neurology and Neurosurgery, Queen’s Square, London, UK, and the **Centre for Biomimetics, School of Construction Management and Engineering, University of Reading, UK. Accepted for publication: 29 September 2008. First published online 13 January 2009. 
middle-ear mechanics by affecting the vibration pattern of the stapes footplate. In the case of electric-acoustic stimulation, it is critical to ensure that middle-ear mechanics are kept in optimal condition, so that acoustic stimulation can be delivered to the lower frequency of the inner ear.

When post-operative, unaided pure tone thresholds were evaluated in patients who received a cochlear implant for the purpose of electricacoustic stimulation, most studies showed threshold elevation at low frequencies. Gantz et al. showed an average $9.5 \mathrm{~dB}$ threshold elevation with their short electrode, whilst Gstoettner et al. showed a similar result with a longer insertion depth (up to $24 \mathrm{~mm}$ ) in 61.9 per cent of their patients. ${ }^{6,7}$ However, 23.8 per cent of the latter group's patients showed larger threshold elevations, ranging from 18 to $40 \mathrm{~dB}$. Both groups regarded a threshold elevation of less than $10 \mathrm{~dB}$ as a good hearing preservation result.

One may speculate that the small threshold elevation was due not to damage in the remaining hair cells but rather to altered middle-ear mechanics caused by increased cochlear impedance as a result of electrode insertion. It has been demonstrated both in animal models and in clinical practice that mechanical injury to the inner ear often leads to catastrophic hearing loss, with a much larger than $10 \mathrm{~dB}$ threshold elevation and in many incidences a 'dead ear'. However, little is known about the effect of cochlear implantation on the displacement pattern of the ossicles and hence on middle-ear mechanics. The aim of this study was to investigate the effect of cochlear implant electrode insertion on the middle-ear, low frequency transfer function, using intra-operative laser Doppler vibrometry.

\section{Patients and methods}

\section{Approvals}

The patient recruitment and method protocols were approved by the National Health Service (NHS) research ethics committee. In addition to ethical approval, research and development approval was obtained from Guy's and St Thomas' NHS Trust. Because laser Doppler vibrometry is not used clinically, the device does not come with the necessary approval from the Medical Devices Agency. The equipment therefore underwent rigorous testing by the medical physics department at Guy's Hospital to ensure that the trust health and safety regulations were met.

\section{Patients and surgery}

Twelve patients were initially recruited for the study, five children and seven adults. Their ages ranged from 18 months to 80 years. Seven patients received a right-sided cochlear implant and five a left-sided device.

The inclusion criterion for the study was simply the requirement for a cochlear implant. The patients selected were 'traditional' cochlear implant patients with severe to profound sensorineural hearing loss. The aetiology of their hearing loss included congenital malformation, meningitis, severe presbyacusis and idiopathic causes. None of the patients were undergoing implantation with a view to combined electrical-acoustic stimulation.

The exclusion criterion for the study was middle-ear disease, as determined by history, otoscopy, tympanometry and intra-operative findings. None of the patients recruited were subsequently excluded.

Informed consent was obtained in all cases.

A standard cochlear implant approach was used, via a retroauricular incision and involving partial mastoidectomy and posterior tympanotomy. All patients were implanted with an Advanced Bionics Clarion device (Advanced Bionics Corporation, California, USA) via the round window. The electrode was fully inserted in all but one case.

\section{Measurement system}

Figure 1 demonstrates the experimental arrangement. The measurement system consisted of a sound delivery and calibration system and an operating microscope mounted laser Doppler vibrometry system. Sound was delivered through an ER-2 earphone (Etymotic Research Inc, Illinois, USA) coupled to a customised ear mould which was inserted into the ear canal. The output was calibrated at approximately $3 \mathrm{~mm}$ from the tympanic membrane using an ER-7C probe microphone (Etymotic Research) which was also incorporated within the ear mould with an ER7-14C probe tube (Etymotic Research). The ER-7C probe microphone was calibrated against a standardised calibrator at the beginning of each experiment.

Each patient had an individually tailored earpiece fashioned to hold the earphone and microphone ( $\mathrm{N}$ Donnelly et al., unpublished data). This was made prior to general anaesthetic (GA) in adult patients and under GA in children. The mould was fashioned from the impression material used to make hearing aid moulds and the technique used was the same. A punch biopsy instrument made a hole through the mould large enough to accommodate the tubing for the loud speaker and the microphone. The tubing was positioned through the mould such that the microphone was approximately $3 \mathrm{~mm}$ from the tympanic membrane. The tubing was then secured in position with glue. After the mould was placed in the ear, the speaker and microphone were attached to the tubing and the equipment tested.

The sound stimulus was digitally synthesised using Vibsoft software (Polytec Gmb-H, Waldbronn, Germany) and was exported to a digital-analogue converter. A multisine stimulus was used consisting of tones at 31 different frequencies at $250 \mathrm{~Hz}$ intervals, from $500 \mathrm{~Hz}$ to $8 \mathrm{kHz}$, with each frequency having an intensity of $80 \mathrm{~dB}$ SPL. ${ }^{8}$ A commercial HLV-1000 laser Doppler vibrometry system (Polytec Gmb-H, Waldbronn, Germany) was mounted over the lens of an operating microscope and was used to measure stapes velocity at the stapes head. The helium-neon laser beam was focused on the head of the stapes through the posterior tympanotomy. Intra-operative 


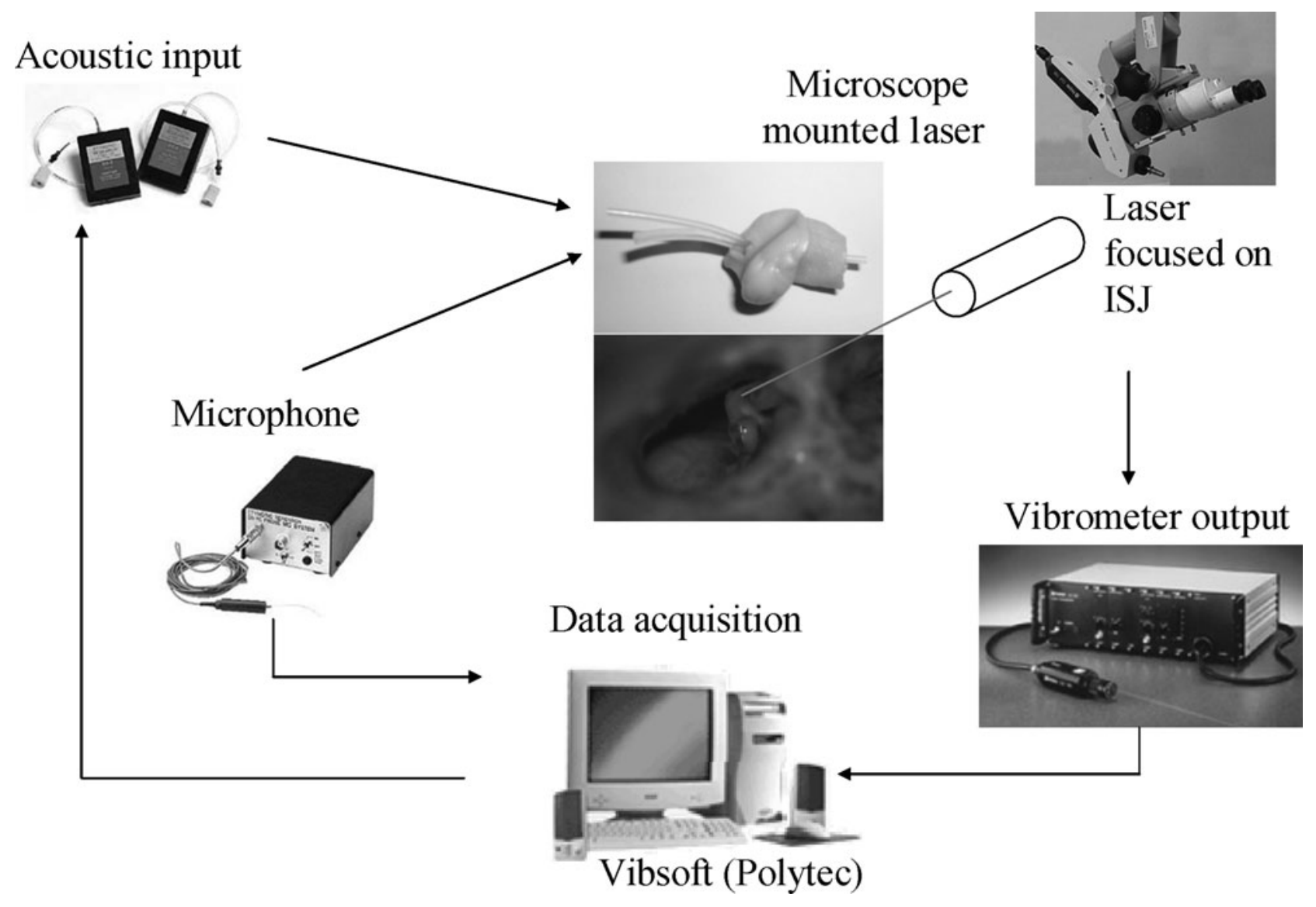

FIG. 1

Arrangement of experimental equipment. ISJ = incudostapedial joint

measurements were made before and after electrode insertion. The reflected signal was detected and decoded by the vibrometer controller to produce an output voltage proportional to the stapes velocity. We concentrated on change in stapes displacement at 250, 500, 1000 and $2000 \mathrm{~Hz}$. The responses obtained prior to electrode insertion were regarded as the baseline reading for each individual. The decibel difference between the pre-implant baseline reading and the postimplant displacement measurement was calculated for each patient. A signal to noise ratio of $15 \mathrm{~dB}$ was set to exclude measurements. Differences of less than $10 \mathrm{~dB}$ between the two measurements were regarded as insignificant, in order to take into account methodological factors such as alteration of the laser angle of the measurement position.

\section{Results}

No results were obtained for five patients. There was hardware failure in one case, displacement of the ear mould in two cases, corruption of data in one case and failure of implantation in one case due to severe ossification of the scala tympani.

As mentioned previously, we were interested in the differences between the pre- and post-implant measurements at frequencies below $2000 \mathrm{~Hz}$. These observed differences were divided into three groups and plotted as bar graphs. The first group (Figure 2) comprised patients showing an increase in stapes displacement after electrode insertion. The second group (Figure 3) comprised patients showing a decrease in displacement after electrode insertion, while the third group (Figure 4) consisted of those showing little change. Figure 2 shows that patients 10 and 11 demonstrated an increase in stapes displacement following insertion of the cochlear implant electrode. This increase in displacement was extremely large in patient 11 , being greater than $30 \mathrm{~dB}$ at all four frequencies. The increase in displacement for patient 10 was approximately half that for patient 11. Figure 3 illustrates the decrease in stapes displacement following cochlear implantation seen in patients eight and 12 . The magnitude of change was very similar for both patients, i.e. a 20-30 dB decrease in displacement. The greatest effect is observed at $1000 \mathrm{~Hz}$. Figure 4 shows findings for patients four, five and seven; i.e. the dB displacement differences between pre- and post-implant recordings were less than $10 \mathrm{~dB}$ and thus deemed insignificant.

\section{Discussion}

This current study is, to the best of our knowledge, the first to measure stapes movement using 


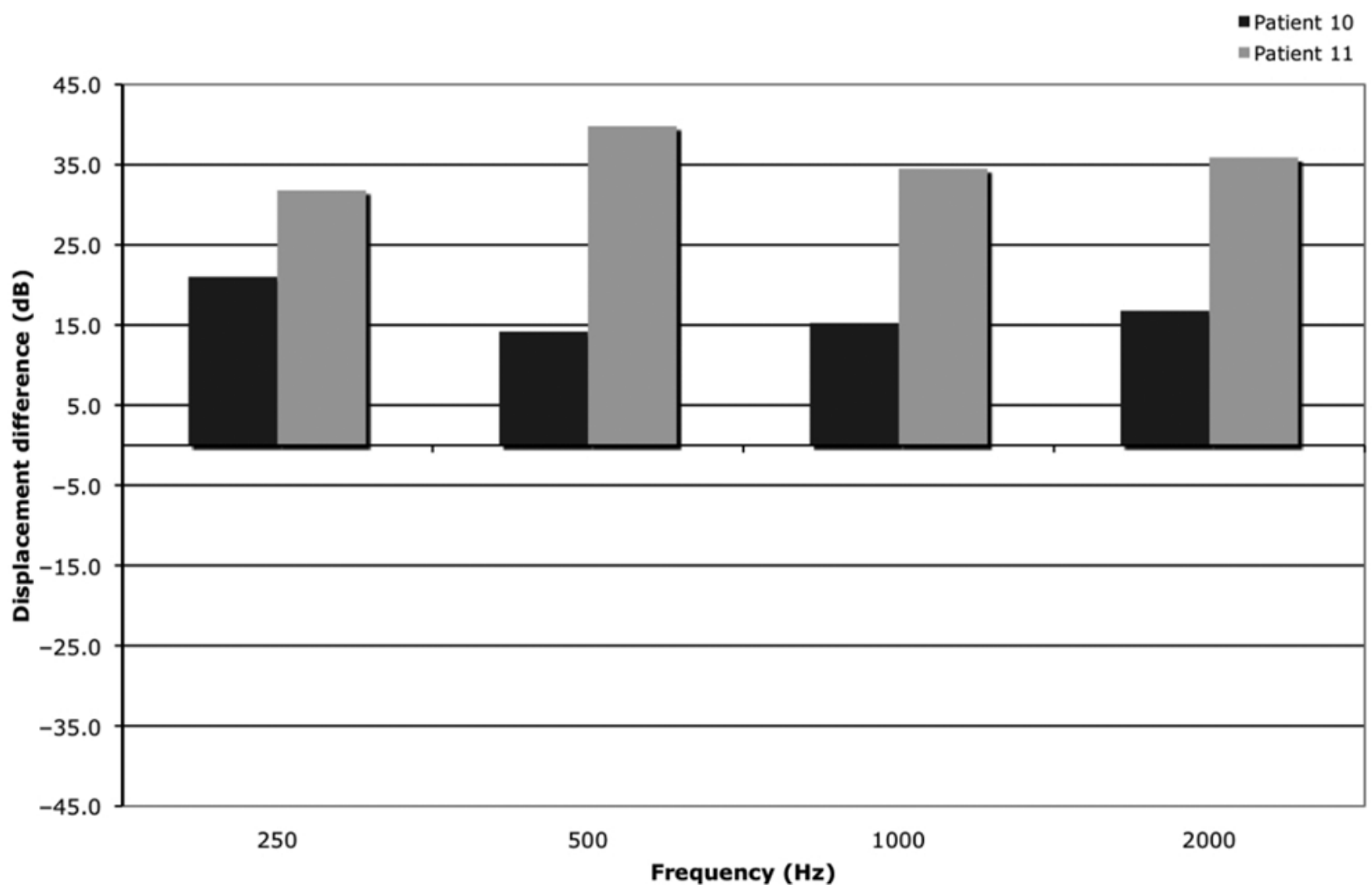

FIG. 2

Stapes displacement results for patients demonstrating increased stapes displacement following cochlear implant electrode insertion.

intra-operative laser Doppler vibrometry following cochlear implantation. A number of in vitro studies have reported the use of laser Doppler vibrometry to study middle-ear transfer function in temporal bones. ${ }^{9-12}$ Huber et al. examined intra-operative stapes movement in seven patients with profound, bilateral hearing loss undergoing cochlear implantation prior to electrode insertion, but no post-implant measurements were taken. ${ }^{13}$ The stapes displacement results obtained were comparable to findings in cadaveric temporal bone preparations but of lower magnitude. Chien et al. have proposed that, with the proper measurement angle corrections, stapes velocity in live and cadaveric ears is similar. ${ }^{14,15}$

The principal finding of this study was the wide variability in the effect of cochlear implantation on stapes displacement. Two patients experienced large increases in their stapes displacement after electrode insertion, while a further two experienced large decreases. In the remaining three patients, the observed changes were not regarded as significant as the magnitude of difference fell within a range that might be explained by methodological factors. It is likely that the changes in stapes displacement observed in some patients would impact on any residual low frequency hearing.

Both the act of electrode insertion and the associated suction required will lead to loss of perilymph from the inner ear. When the cochlea is opened and the implant electrode inserted, it is possible for small air bubbles to enter the scala tympani and for more perilymph to be lost. We can speculate that the variability of our results largely reflects operative variability. This assertion is supported by examination of the literature.

Aibara et al. measured middle-ear transfer function before and after opening the inner ear, in an experimental temporal bone model. ${ }^{10}$ They made a $1.4 \mathrm{~mm}$ diameter opening into the scala vestibule, and were able to prevent air bubbles entering the inner ear by submerging their bone in saline while making the cochleostomy. They demonstrated that merely opening the inner ear, without loss of perilymph, had no significant effect on the stapes velocity transfer function.

Research by Murakami et al. investigated the effect on middle-ear mechanics of altering inner-ear pressure. ${ }^{16}$ They found that changing the inner-ear pressure had an effect on the static displacement of the ossicles, with outward displacement when inner-ear pressure was increased and inward displacement when it was decreased. This effect was greater for positive ear pressure changes than negative ones. Murakami et al. also assessed the vibration velocity of the stapes at four different inner-ear pressures, and found that as inner-ear pressure increased, the amplitude of the stapes velocity decreased. The increased inner-ear pressure damped the stapes vibration, with the effect being greatest at lower frequencies. 


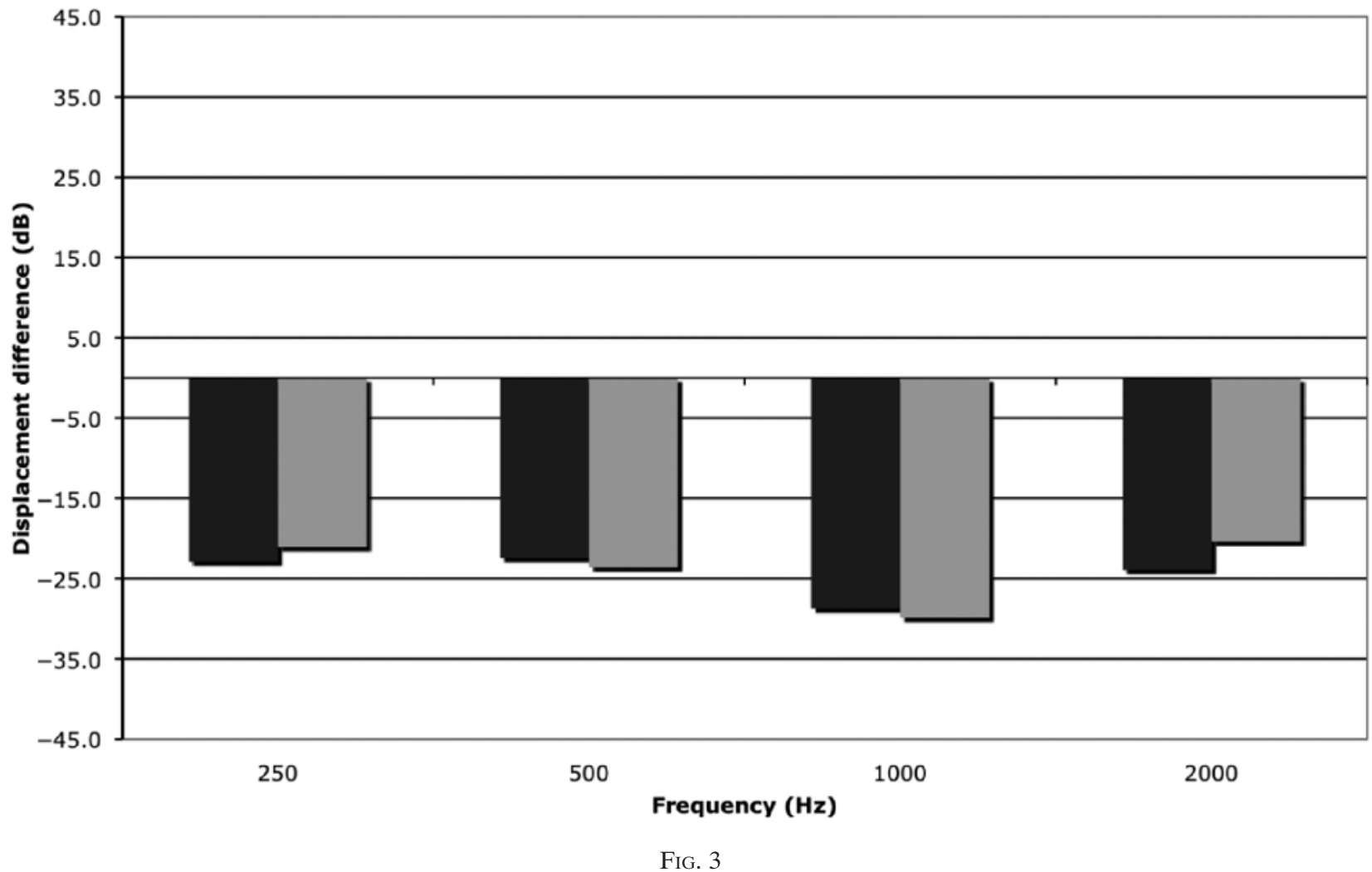

Stapes displacement results for patients demonstrating decreased stapes displacement following cochlear implant electrode insertion.

- This study aimed to investigate the impact of cochlear implant electrode insertion on middle-ear low frequency function in humans

- Stapes displacement was measured in seven patients undergoing cochlear implantation

- Insertion of a cochlear implant electrode into the scala tympani had a variable effect on stapes displacement

- Insertion of a cochlear implant electrode produced a change in stapes displacement at low frequencies, which may have an effect on residual low frequency hearing thresholds

The insertion of a cochlear implant electrode into the scala tympani is likely to result in an increase in inner-ear pressure, particularly if little perilymph is lost. This finding may explain the decrease in stapes velocity observed in two of our patients. A number of studies have investigated the effect on stapes displacement of draining the cochlea. Gunderson and Høgmoen found that the vibratory amplitude of the stapes was larger after cochlear fluid had been drained. ${ }^{17}$ Gyo et al. investigated stapes head displacement before and after draining the cochlea, in one temporal bone; they found that draining the cochlea resulted in an increased stapes displacement of approximately $10 \mathrm{~dB}$ at $1000 \mathrm{~Hz}^{18}$ Lord et al. assessed the effects of draining cochlear fluids on stapes displacement in human temporal bones. ${ }^{19}$ They measured displacement frequency characteristics in five bones and found that the displacement response was less in the drained cochlea at low frequencies. These findings contradicted the results predicted by their own electrical analogue model (which predicted that, in a drained cochlea, stapes displacement would increase by over $10 \mathrm{~dB}$ at all frequencies, with the largest increase being observed at $1000 \mathrm{~Hz}$ ). Gan et al. also examined the effect of draining the cochlea in six temporal bones. ${ }^{11}$ They found an increase in stapes footplate and incudostapedial joint displacement after drainage; however, these changes mainly affected frequencies greater than $1000 \mathrm{~Hz}$. Their own finite element model predicted an increase in stapes displacement (after cochlea drainage) beyond $500 \mathrm{~Hz}^{20}$ They concluded that draining the cochlea resulted in an increase in stapes displacement as a result of reduced cochlear impedance. We can speculate that the increase in stapes displacement that we observed in two of our patients may have been due to decreased cochlear impedance as a result of perilymph loss.

Ultimately, the exact effect of cochlear implant insertion on cochlear impedance is unknown. We hypothesise that the volume effect of a cochlear electrode implanted within the scala tympani would increase cochlear impedance, while perilymph loss would reduce impedance. If the pressure increase 


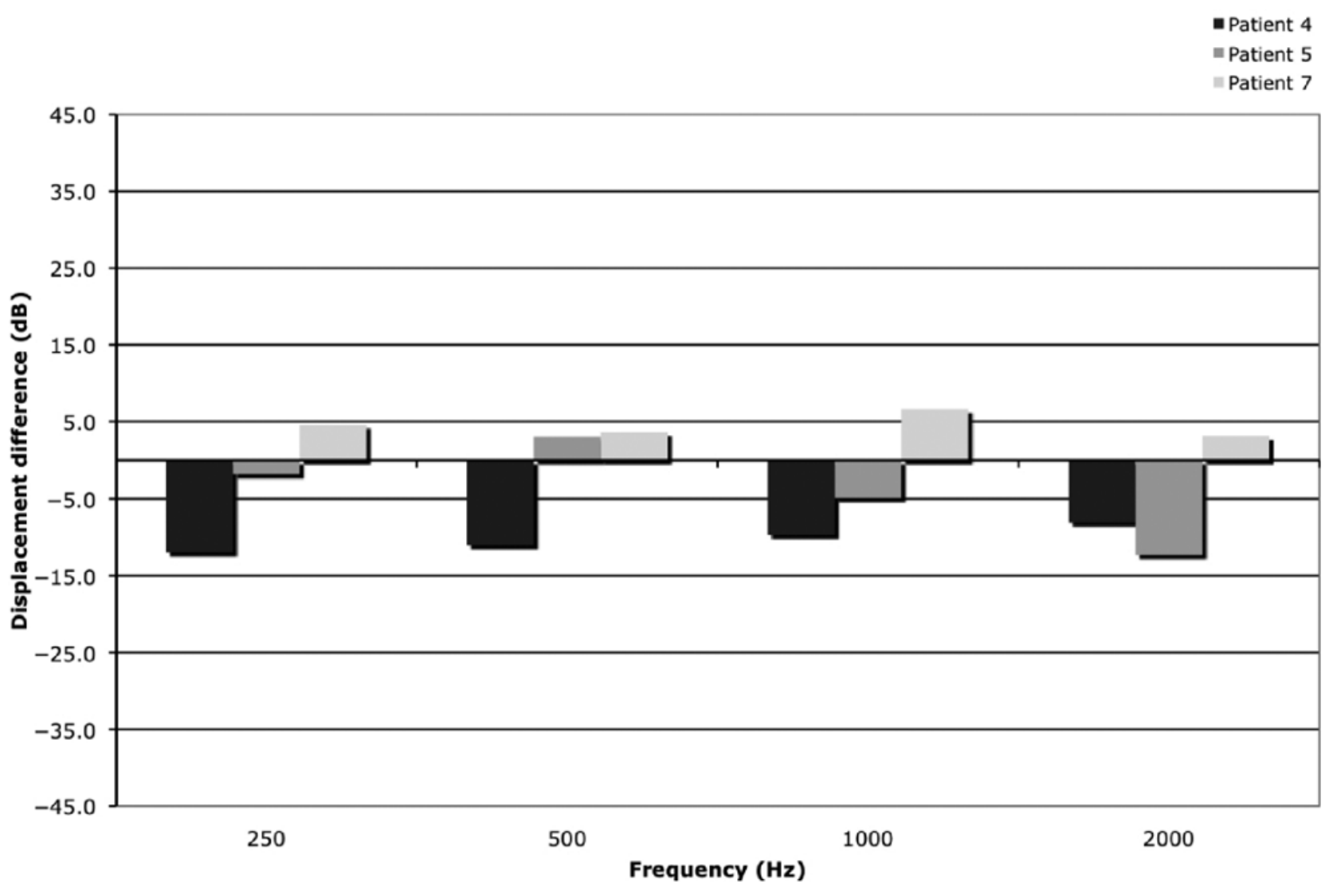

FIG. 4

Stapes displacement results for patients demonstrating unchanged stapes displacement following cochlear implant electrode insertion.

and perilymph loss were balanced, there would be little change in the impedance. Increased cochlear impedance would result in decreased stapes displacement, while decreased cochlear impedance would result in increased stapes displacement. Displacement would remain relatively unchanged if the above effects balanced out to leave cochlear impedance unchanged. This hypothesis provides a mechanism with which to explain the variability of our results.

Our findings raise the question of the effect of altered stapes displacement on the residual low frequency hearing of a patient who has undergone cochlear implantation with a view to combined electrical and acoustic stimulation. It is possible to speculate that any large decrease in stapes displacement would have a detrimental effect on the low frequency thresholds. It is uncertain how long any effects related to altered cochlear impedance would last. One would expect perilymph homeostasis to redress most of the changes following cochlear implant insertion. It is known that when the bony otic capsule is opened or a fluid sample is withdrawn, any lost perilymph is rapidly replaced by a flow of cerebrospinal fluid entering the scala tympani through the cochlear aqueduct. ${ }^{21}$ However, it is possible that there will be some permanent alteration in stapes displacement, and this may explain the audiometric threshold shifts observed by groups investigating patients receiving combined electricalacoustic cochlear stimulation.
Further research is required to answer these questions. One approach would be to repeat our experiments in patients receiving a cochlear implant with a view to both electrical-acoustic stimulation, and to test for correlation between the observed change in stapes displacement and any changes in patients' low frequency audiometric thresholds.

\section{Conclusion}

We have demonstrated that the insertion of a cochlear implant electrode can have an effect on stapes displacement. The observed effect was variable, and we have discussed the possible reasons for this. It is likely that some of the changes observed would affect residual low frequency hearing in patients receiving cochlear implants allowing combined electrical-acoustic stimulation. We have also demonstrated that in vivo laser Doppler vibrometry measurements are both possible and practical in a surgical environment, without putting the patient at unnecessary risk. During this study, we have been able to refine the methodological procedures required for this type of research, which differ in many ways from laboratory-based temporal bone work. Laser Doppler vibrometry, apart from being an important tool in middle-ear research, is likely to assume a greater role in the in vivo setting, providing valuable information about surgical interventions. 


\section{Acknowledgement}

This research was funded by Guy's Hospital Charitable Foundation Trust.

\section{References}

1 Gantz BJ, Turner CW. Combining acoustic and electrical hearing. Laryngoscope 2003;113:1726-30

2 Turner CW, Gantz BJ, Vidal C, Behrens A, Henry BA. Speech recognition in noise for cochlear implant listeners: benefits of residual acoustic hearing. J Acoust Soc Am 2004;115:1729-35

3 von Ilberg C, Kiefer J, Tillein J, Pfenningdorff T, Hartmann R, Sturzebecher E et al. Electric-acoustic stimulation of the auditory system. New technology for severe hearing loss. ORL J Otorhinolaryngol Relat Spec 1999;61:334-40

4 Kiefer J, Gstoettner W, Baumgartner W, Pok SM, Tillein J, Ye $\mathrm{Q}$ et al. Conservation of low-frequency hearing in cochlear implantation. Acta Otolaryngol 2004;124:272-80

5 Kiefer J, Pok M, Adunka O, Sturzebecher E, Baumgartner W, Schmidt M et al. Combined electric and acoustic stimulation of the auditory system: results of a clinical study. Audiol Neurotol 2005;10:134-44

6 Gantz BJ, Turner CW, Gfeller KE, Lowder MW. Preservation of hearing in cochlear implant surgery: advantages of combined electrical and acoustical speech processing. Laryngoscope 2005;115:796-802

7 Gstoettner W, Kiefer J, Baumgartner WD, Pok S, Peters S, Adunka O. Hearing preservation in cochlear implantation for electric acoustic stimulation. Acta Otolaryngol 2004; 124:348-52

8 Huber AM, Schwab C, Linder T, Stoeckli SJ, Ferrazzini M, Dillier N et al. Evaluation of eardrum laser Doppler interferometry as a diagnostic tool. Laryngoscope 2001;111:501-7

9 Voss SE, Rosowski JJ, Merchant SN, Peake WT. Acoustic responses of the human middle ear. Hear Res 2000;150:43-69

10 Aibara R, Welsh JT, Puria S, Goode RL. Human middle-ear sound transfer function and cochlear input impedance. Hear Res 2001;152:100-9

11 Gan RZ, Wood MW, Dormer KJ. Human middle ear transfer function measured by double laser interferometry system. Otol Neurotol 2004;25:423-35

12 Needham AJ, Jiang D, Bibas A, Jeronimidis G, Fitzgerald O'Connor A. The effects of mass loading the ossicles with a floating mass transducer on middle ear transfer function. Otol Neurotol 2005;26:218-24
13 Huber A, Linder T, Ferrazzini M, Schmid S, Dillier N, Stoeckli S et al. Intraoperative assessment of stapes movement. Ann Otol Rhinol Laryngol 2001;110:31-5

14 Chien W, Ravicz ME, Merchant SN, Rosowski JJ. The effect of methodological differences in the measurement of stapes motion in live and cadaver ears. Audiol Neurotol 2006;11:183-97

15 Chien W, Rosowski JJ, Ravicz ME, Merchant SN. Measurements of stapes velocity in live human ears. $29^{\text {th }}$ Midwinter research meeting of the Association for Research in Otolaryngology. 2006. Abstract 641

16 Murakami S, Gyo K, Goode RL. Effect of increased inner ear pressure on middle ear mechanics. Otolaryngol Head Neck Surg 1998;118:703-8

17 Gunderson T, Høgmoen K. Holographic vibration analysis of the ossicular chain. Acta Otolaryngol 1976;82: $16-25$

18 Gyo K, Aritomo H, Goode RL. Measurement of the ossicular vibration ratio in human temporal bones by use of a video measurement system. Acta Otolaryngol 1987;103: $87-95$

19 Lord RM, Abel EW, Wang Z. Effects of draining cochlear fluids on stapes displacement in middle-ear models. $J$ Acoust Soc Am 2001;110:3132-9

20 Gan RZ, Sun Q, Dyer RK. Three-dimensional modelling of middle ear biomechanics and its application. Otol Neurotol 2002;23:271-2

21 Mynatt R, Hale SA, Gill RM, Plontke SK, Salt AN. Demonstration of a longitudinal concentration gradient along the scala tympani by sequential sampling of perilymph from the cochlear apex. JARO 2006;7:182-93

Address for correspondence:

Mr A Fitzgerald O'Connor,

Department of Otolaryngology, Head and Neck Surgery,

St Thomas' Hospital,

Lambeth Palace Road,

London SE1 7EH, UK.

E-mail: afoc@globalnet.co.uk

Mr A Fitzgerald O'Connor takes responsibility for the integrity of the content of the paper.

Competing interests: None declared 